\section{Severe brimonidine eye drop intoxication in a neonate as an accidental oral ingestion}

\author{
Musa Silahli* \\ Department of Pediatrics, Division of Neonatology, Sivas Government Hospital, Sivas, Turkey
}

\section{Abstract}

Brimonidine tartrate eye drops are a topical agent used to treat glaucoma in children over 2 years of age and adults. It is banned for children younger than 2 years of age because postmarketing studies have shown serious side effects. Colic is common in infants, which worries parents. And parents often use herbal and chemical medicines to solve this problem. We present a 12-day-old newborn with brimonidine eye drop intoxication, in which the drug was mistakenly administered orally to treat the colic problem.

\begin{abstract}
More Information
*Address for Correspondence: Musa Silahli, MD, Sivas Government Hospital, Yesilyurt Mh. Şifa Cd. No: 4 Center, Sivas, Turkey, Email: msilahli@gmail.com

Submitted: December 07, 2021

Approved: January 03, 2022

Published: January 04, 2022

How to cite this article: Silahli M. Severe brimonidine eye drop intoxication in a neonate as an accidental oral ingestion. J Adv Pediatr Child Health. 2022; 5: 001-003.
\end{abstract}

DOI: 10.29328/journal.japch.1001044

Copyright License: (๑) 2022 Silahli M. This is an open access article distributed under the Creative Commons Attribution License, which permits unrestricted use, distribution, and reproduction in any medium, provided the original work is properly cited.

D) Check for updates

O open access

\section{Introduction}

Topical eye drops are commonly used in the pediatric population for the treatment of various ocular diseases. Some of them may have serious systemic effects [1,2]. Eye drops containing brimonidine tartrate are used to lower intraocular pressure. Brimonidine is an alpha- 2 adrenergic receptor agonist that is 1000 -fold more selective for the alpha-2 adrenoceptor than the alpha- 1 adrenoceptor. This selectivity results in no mydriasis and the absence of vasoconstriction in microvessels associated with human retinal xenografts. Brimonidine eye drops are thought to work by reducing the amount of fluid that you make in the front part of your eye (called aqueous humour) and by increasing the drainage of fluid from your eye. These two actions help to lower the pressure within your eye. Side effects of brimonidine include sedation, mediated by postsynaptic receptors located in the locus coeruleus, behind the blood-brain barrier; hypotension and bradycardia, which are thought to be related to both central and peripheral actions [7]. Coma, respiratory depression and miosis are central effects mediated by alpha- 2 receptor stimulation. Theoretically, since it is a more lipophilic agent than clonidine, it is expected to be less neurotoxic, but severe neurotoxicity is observed in newborns, probably because the blood-brain barrier is less mature [6].

This case report describes a neonate who had accidentally taken briminidone orally from his mother, resulting in severe central nervous system depression with shallow breathing.

\section{Case presentation}

A 12-day previously healthy newborn was admitted to our pediatric emergency department with hypotension, respiratory changes, and lethargy. Prenatal, natal, and early postnatal examinations were unremarkable. Two days earlier, a pediatrician prescribed her some medications, including lactase enzymes and probiotics, for colic pain. While taking the history, it was recorded that the grandmother gave the glaucoma eye drops that she used in her glaucoma treatment, orally, to her grandchild 20 minutes before she called the pediatric emergency service. The child was brought in by her parents complaining of irregular breathing and pale skin. She was immediately admitted to the neonatal intensive care unit (NICU). Physical examination in the NICU revealed that she was hypotensive, lethargic, irregular breathing and unresponsive to pain which were associated with central nervous system depression. Her body weight was $3900 \mathrm{~g}$. She did not have dysmorphic findings. Vital signs were as follows: Body temperature: 36.7 ; respiratory rate: 18/min (irregular); heart rate: 125 beats/min; blood pressure: $87 / 37$ (51) mm Hg. Capillary refilling time was 2 seconds. Peripheral pulses and femoral pulses were well palpable. The anterior fontanel was normal. Ocular examination revealed bilateral miosis. Irregular and shallow breathing without rales or wheezing was noted (Video 1). There were no cardiovascular abnormal clinical findings. Neurologic examination revealed hypotonia, lethargy, and decreased deep tendon reflex with insensitivity to pain. 


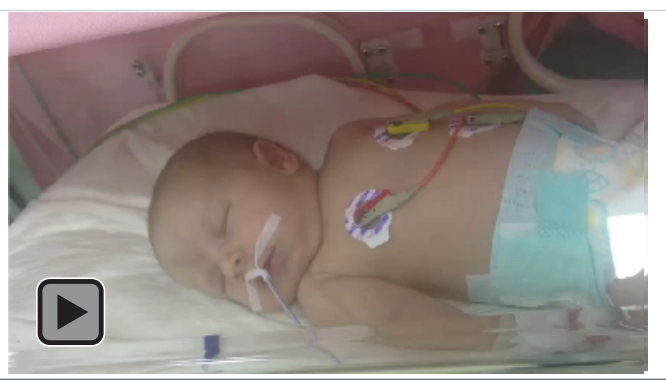

Video 1: This video shows severe central nervous system depression.

Laboratory values were unremarkable. Blood count, serum electrolytes, renal function, liver function, and blood glucose were within the normal range during the observation period. Plasma ammoniac level was $48 \mathrm{micmol} / \mathrm{L}$. Blood level of brimonidine could not be measured as it was not available. Urine or serum toxicology screens were not performed to rule out other intoxications due to unavailability. Meningitis was not considered and lumbar puncture was not performed because infection markers were negative and clinical signs were associated with an inadvertent drug. Blood culture was negative. Chest radiograph showed consolidation of the right upper lung, which was considered to be pneumonia (Figure 1).

This was thought to be associated with severe cerebral depression. Blood gas analysis showed mild respiratory acidosis as follows pH: 7.31; pCO2: $54.3 \mathrm{mmHg}$; 02 : $42.4 \mathrm{~mm}$ Hg; HCO3: 27 mmol/L; BE: -0.6 mmol/L; lactate: $1.64 \mathrm{mmol} / \mathrm{L}$.

The patient was admitted to the NICU with brimonidine intoxication and pneumonia. She received intravenous fluids, supportive care, and was not treated with medications for intoxication except for pneumonia. Since she was hypoactive and hypotonic, she was not fed for 24 hours due to the risk of aspiration. After 24 hours, feeding was gradually increased via an orogastric tube. Full enteral feeding was started in 48 hours and intravenous fluid was stopped. Hypotension, shallow breathing, and neurologic abnormalities improved after 48 hours with full recovery of vital signs. Up to 48 hours clinical signs improved. Heart rate was $150 / \mathrm{min}$, blood pressure was 74/52 $\mathrm{mm} \mathrm{Hg}$, respiratory rate was 43/ minute with regular breathing, body temperature was $36.7^{\circ} \mathrm{C}$, and capillary refilling time was 3 seconds. The respiratory effort was good. Hypotonia was resolved, deep tendon reflex

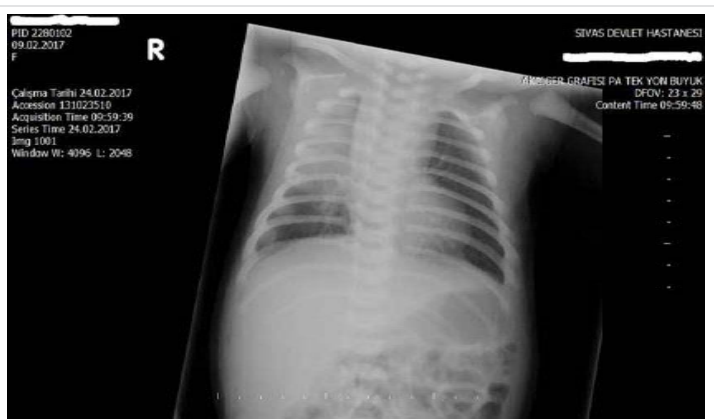

Figure 1: Chest $\mathrm{X}$-ray shows right upper consolidation of the lung. response was normal. Alert response to surrounding sound and light was good, painful stimuli and responses to touch were good. Pupils were isochoric and light response was normal. Clinical improvement was as expected. She was discharged on the $9^{\text {th }}$ day of admission after treatment for pneumonia was completed.

\section{Discussion}

Brimonidine tartrate ophthalmic solution is an antiglaucoma agent that is not recommended for use in pediatric patients younger than 2 years of age. This is because the safety and efficacy of brimonidine tartrate has not been studied in pediatric patients younger than 2 years of age [2]. Studies have shown that brimonidine tartrate has a dual mechanism of action, reducing aqueous humor production and increasing uveoscleral outflow. After ocular administration of a $0.2 \%$ solution, plasma concentrations peaked within 1 to 4 hours and declined with a systemic half-life of approximately 3 hours in human adults [3]. In humans, the systemic metabolism of brimonidine is extensive. It is metabolized primarily by the liver. Excretion of the drug and its metabolites occurs primarily in the urine. In a well-controlled clinical trial conducted in pediatric glaucoma patients aged 2 to 7 years), the most commonly observed adverse effects of $0.2 \%$ brimonidine tartrate ophthalmic solution administered three times daily were drowsiness $(50 \%$ - 83\% in patients aged 2 to 6 years) and decreased attention [3]. The main adverse effects of brimonidine in published articles were adverse effects on the central nervous system and impaired systemic blood flow.

There are several cases of eye drop poisoning or adverse effects of eye drops in the pediatric population in the literature. These cases involve brimonidine, tymolol, and tetrahydrozoline.

These intoxications have been observed with topical use for glaucoma treatment, topical use for ulcerated hemangiomas, and oral nasal ingestion by accident $[8,9]$. Kriyazov, et al. has described severe central nervous system depression in two pediatric cases in whom brimonidine eye drops had adverse effects when used topically to treat congenital glaucoma [4]. One of them needed positive inotropic support to maintain blood pressure; the other needed only conservative treatment. In our patient, mechanical ventilation and positive inotropic support were not required.

Due to its highly lipophilic nature, brimonidine readily passes the blood-brain barrier, which increases the risk for the occurrence of adverse effects in the central nervous system. In previous studies, central nervous system depression was found to be more pronounced in younger children.

The other reason for this could be related to the lower circulating blood volume and immature blood-brain barrier. In a questionnaire-based parent survey evaluating the adverse effects of brimonidine eye drops, the authors found 
that adverse effects of brominidine were common. They recommended that brimonidine should be avoided in children weighing less than $20 \mathrm{~kg}$ and in children younger than 6 years $[5]$.

In the study by Becker, et al. it was a retrospective study based on the American Association of Poison Control Centers' Toxic Exposure Surveillance System database and the US Food and Drug Administration's Medwatch Adverse Events Reporting System, the maximum age of poisoning was approximately 2 years [6]. In the literature which was investigated the use of naloxone as an antidote, despite the existence of several reports on its efficacy, routine use as an antidote for brimonidine intoxication is not recommended. The clinical symptoms are usually mild and/or temporary. Intensive monitorization, as was the case of such patients, is usually enough. Indications for the use of naloxone in brimonidine poisoning remain uncertain [6]. The lowest dose is not known, but poisonings have been reported even at doses such as 2 or 3 drops $[8,9]$.

We described this case report to draw attention to the fact that opthalmic preparations, including brimonidine in particular, can be very dangerous to children, especially in neonates. This case was presented to illustrate how brimonidine intoxication is treated and the clinical features of brimonidine intoxication in a newborn. And it is also quite difficult to distinguish some colic drops from brimonidinecontaining eye drops (Figure 2). There must be a warning on the brimonidine-containing eye drops that they are very dangerous for children.

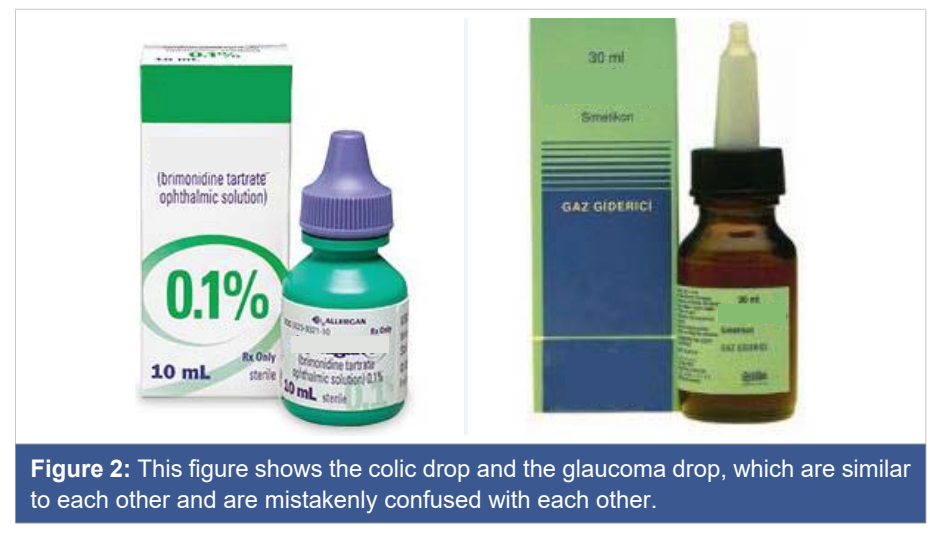

\section{References}

1. Levy $Y$, Zadok D. Systemic side effects of ophthalmic drops. Clin Pediatr (Phila). 2004; 43: 99-101.

PubMed: https://pubmed.ncbi.nlm.nih.gov/14968900/

2. Gray C. Systemic toxicity with topical ophthalmic medications in children. Paediatr Perinat Drug Ther. 2006; 7: 23-29.

3. Alphagan P. Irvine, CA: Allergan Incorporated; 2007.

4. Kiryazov K, Stefova M, lotova V. Can ophthalmic drops cause central nervous system depression and cardiogenic shock in infants? Pediatr Emerg Care. 2013; 29: 1207-1209.

PubMed: https://pubmed.ncbi.nlm.nih.gov/24196091/

5. Al-Shahwan S, Al-Torbak AA, Turkmani S, et al. Side-Effect profile of Brimonidine Tartrate in Children. Ophthalmology. 2005; 12: 2143-2148. PubMed: https://pubmed.ncbi.nlm.nih.gov/16225927/

6. Lai Becker M, Huntington N, Woolf AD. Brimonidine tartrate poisoning in children: frequency, trends, and use of naloxone as an antidote. Pediatrics. 2009; 123: 305-311.

PubMed: https://pubmed.ncbi.nIm.nih.gov/19124581/

7. Bowman RJC, Cope J, Nischal KK. Ocular and systemic side effects of brimonidine 0.2\% eyedrops(Alphagan $囚$ )inchildren.Eye. 2004;18:24-26. PubMed: https://pubmed.ncbi.nlm.nih.gov/14707960/

8. Ghaffari Z, Zakariaei Z, Ghazaeian M, Jafari R, Ezoddin N, et al. Adverse effects of brimonidine eye drop in children: A case series. $J$ Clin Pharm Ther. 2021; 46: 1469-1472.

PubMed: https://pubmed.ncbi.nlm.nih.gov/33626597/

9. Deslandes G, Bouquié R, Boels D, Grégoire M, Grison-Hernando H, et al. Accidental poisoning with brimonidine eye drops in a 9-day-old infant. Toxicologie Analytique et Clinique. 2015; 27: 14-17. 